\title{
Work that Enables Care: Understanding Tasks, Automation, and the National Health Service
}

\author{
Matt Willis ${ }^{[0000-0002-9120-7319]}$ and Eric T. Meyer ${ }^{[0000-0002-1998-7162]}$ \\ University of Oxford, Oxford, UK \\ [matthew.willis], [eric.meyer] @oii.ox.ac.uk
}

\begin{abstract}
Automation of jobs is discussed as a threat to many job occupations, but in the UK healthcare sector many view technology and automation as a way to save a threatened system. However, existing quantitative models that rely on occupation-level measures of the likelihood of automation suggest that few healthcare occupations are susceptible to automation. In order to improve these quantitative models, we focus on the potential impacts of task-level automation on health work, using qualitative ethnographic research to understand the mundane information work in general practices. By understanding the detailed tasks and variations of information work, we are building a more complete and accurate understanding of how healthcare staff work and interact with technology and with each other, often mediated by technology.
\end{abstract}

Keywords: automation, primary care, ethnography, sociotechnical

\section{Introduction}

What do we mean when we talk about the automation of jobs? If we are to believe the most hyperbolic of public commentators, we face a near future where 'robots are coming for our jobs' and human labor is made largely redundant except in support of a large, automated, robotic workforce [1,2]. Of course, this concern about being replaced by robots is nothing new: Time Magazine carried a cover in 1980 predicting "The Robot Revolution"[3], and similar examples have occurred periodically in media outlets going back as far as 200 years [4]. However, the rhetoric has exceeded the reality: Bessen (2016) argues only one occupation has been completely eliminated by automation over the last 60 years (namely, elevator operators) [5].

Realistically, these visions (or fears, depending on your point of view) of an automated workforce will be much more nuanced in practice in the future just as they have been in the past. Some jobs will almost certainly become fully or nearly fully automated, while others will see human labor increasingly augmented either by computers (and algorithms) or by robotic machines. Recent advances in the areas of machine learning, machine vision, computational statistics, data mining, various artificial intelligence methodologies, and mobile robotics, were made possible from large investments in both the public and private sector. Some of these technical advances are starting to see practical applications. 
Thus, we need to ask ourselves not 'will there be widespread automation in the coming years' but instead we need to examine the ways that analogue and manual modes of work are transformed when individuals and organizations shift to digital and (partially) automated ways of working. This paper reports on work-in-progress to better understand these more nuanced shifts to digital work and automation of tasks in a single setting: the UK health sector.

\section{Background: Automation and the state of NHS primary care in England}

In a widely publicised 2013 working paper (since published in 2017) Frey and Osborne analyzed a dataset from the U.S. Department of Labor called O*NET [6]. The O*NET data captures various measures about the nature of work and the skills needed for occupational work activities. The goal of their analysis was to understand how susceptable jobs are to automation technologies given advances in machine learning and related artificial intelligence and robotic technologies. The conclusion Frey and Osborne draw from analyzing 702 detailed occupations is that about 47 percent of U.S employment is susceptable to different levels of automation [7, 8]. The Frey and Osborne study is but one in many that show technologies substituting for human labor and creating widespread unemployment [9-13].

One particular pattern in the Frey and Osborne analysis is that most healthcare occupations featured a remarkably low probablity for computerisation. This is, in part, due to the fact that most healthcare occupations contain features that are considered current bottlenecks to computerisation, including social intelligence, social perceptiveness, negotiation, persuasion, caring for others, origionality, and creativity. The other part of the low computerisation probability for healthcare professions is because the empirical data available was at an aggregated level. Fine-grained data on the detailed work practices and skills needed to complete individual tasks and sub-tasks do not exist at any large scale, thus, we have a poor understanding of how future automation and artificial intelligence research may impact complex work arrangements that involve many heterogenous and non-repetitive tasks, as in the healthcare sector.

While automation may threaten vulnerable workers in many economic sectors, healthcare is different in that it is one of the few sectors where automation is seen by leaders, experts, policy makers, and workers as an opportunity to deal with a potential crisis. The potential crisis is particularly acute the NHS in England, where the primary care system faces staff shortages, increased workloads, increased service demand, reduced budget, skill shortages and decreased time for patient consultations in general practice services [14-16]. The reasons for the current state of NHS primary care are complex and multi factorial, the way out of this is seen to require innovation in areas such as service delivery, use of technology, staff training and patient education. One path toward relieving pressure on NHS primary care staff is the use of automation as a force multiplier, making it possible to get more done with less effort. This presents a challenge to understand where and to what extent automation can be developed, de- 
ployed and implemented into the work practices in the primary care setting. Furthermore, many technologies are developed and implemented in NHS that become cumbersome burdens to healthcare work, emphasizing the need to understand the social environment and work practices before technological interventions begin.

\section{Empirical setting and analytical methods}

The work presented in this paper is research in development from the initial phase of the project. As we begin to collect and analyze data, we find ourselves in the position of having to classify the work practices and ways people work with technology to accomplish tasks. This granular analysis has led to thinking about how complex tasks are completed, and what information about that task a computer would need to know.

The project team includes a field researcher, who visits primary care practices and shadows every occupational role at each site to understand the work tasks that are performed by each occupation. In addition to this shadowing, we conduct interviews, gather documents, and capture photos and videos to understand how tasks flow through the primary care clinic. These far more detailed qualitative data are then linked to other data about the sector and modeled using methods developed in the original Frey and Osborne work to create a more detailed and nuanced understanding of automation in the primary health care sector in England.

At the time of this writing we have recruited four primary care general practice centers, and plan to work with 6-7 more sites in the coming year. The field researcher spends an average of a week in the field with each site observing each occupational role performing their duties. The goal is to develop an understanding for how each occupation works, what tasks they are responsible for, how they accomplish those tasks, the kinds of technologies needed to accomplish each task, and what aspects of their work rely on other people and how multiple people touch on different aspects of the same task. After gathering these detailed data the field researcher begins to synthesize the tasks across every primary care practice with a sensitization for identifying features of each task that can help to understand properties of task computerization. Qualitative coding methods including grounded theory and constant comparison approaches [7, 8], are used in analysis. The process for grounding quantitative models of task automation probabilities starts with observations of primary care work as previously described. Then, the field researcher analyzes field notes and collected documents using a constant comparative method, creating a matrix of occupations and tasks performed by each task. Once all tasks have been extracted from the data a grounded theory approach is used for qualitatively coding the tasks to develop features or classifications of each task. These features and classifications of work tasks are coded for different properties including the use of technologies, social aspects of tasks, types of interruptions, partially automated parts of the task, and other task classifications that emerge from the data. 


\section{$4 \quad$ Findings}

Our preliminary analysis reveals the size and scope of tasks performed in primary care, indicators of partial automation, and preferences for certain practices. The heavy use of desktop computers is an important indicator for automation because it is likely that software based automation will be some of the first tasks automated through technologies like robotic process automation. We show how the configuration of the same technology in different ways presents a challenge to automation, along with the structure of certain work in primary care.

From our data, we have identified ten unique occupational roles in primary care, and some of those roles can be further divided into sub categories. For example we catalogue GPs as just one role: general practitioner. This role also includes different "ranks" or seniority of GPs such as partner GP, salaried GP, locum GP, duty GP, various levels of training (ST 1, ST3) and so on. For the purposes of this research the tasks they perform are largely the same and the titles indicate acquired knowledge and status. These ten occupations are what make primary care run and comprise a total of 135 regular tasks that we have identified. Every occupation has around 15-20 tasks that they regularly perform. For practice staff (non-clinical occupations) there are around three to eight tasks that require the collaboration of another person in the office to complete the task. This includes tasks like signing off on a prescription or letter, reviewing a document, gathering signatures, or entering in their portion of data into a system that someone else will use. Every task performed relied on a desktop computer unless it was a purely paper based task, a face to face meeting, or a phone call.

Each primary care center is home to a long list of medical technologies: needles, otoscope, stethoscope, dermascope, reflex hammer, thermometer, blood pressure meter, scale, electrocardiogram machine, and so on. However, the technology at the centre of every primary care clinic is the desktop computer. These desktop computers necessitate an ever longer list of software that enables the primary care clinic to run. Furthermore, the software that runs on every desktop computer is often configured very differently from practice to practice. The electronic medical record (EMR) that runs on every desktop computer is what all staff in primary care spend the majority of their time interacting with. Every EMR has the ability to keep and share tasks. The task EMR functionality is an analogy for a "to do" list: users create a task, add some information about the task, and it shows on their task list in the medical record. The use of this one feature varied across the three initial field sites. One site chose not to use tasks at all due to the demand of senior GPs in favor of a paper based system where receptionists would stamp (rubber stamp with ink) meta data that amounted to different tasks directly on paper documents. These paper documents then moved around the clinic in a specific order. Another clinic, on the other hand, used tasks for everything, even going so far as to communicate through task comments between GPs and practice staff. The third field site used a mix of both systems observed in the previous two sites: mostly digital tasks with specific tasks and workflows having a requirement to be paper based. Both sites that saw the use of tasks also developed a very specific strategy for tasks. The intended use of tasks is to send them to other people, similar to an email, and have them appear on a "to do" list for the recipient. In both cases GPs would also create tasks for themselves and then send them to their self. This data from EMR task use is important because it is a prime example of the configuration of technologies and software that the 
primary care practice to achieve certain goals using the experience and knowledge they have. It also shows that one simple technology, tasks, can be configured in at least three different ways from its intended purpose. This reconfiguration is a form of social shaping of technology, as the technology can either be shaped by use or (in the case of a preference for paper) by non-use, avoidance, or circumvention.

Our last finding to emerge from a preliminary analysis are three directions of transactional communication that occur at each primary care practice. They are communication within the practice, communication outside the practice, and communication directly to the patient. Communication within the practice includes tasks like the aforementioned tasks functionality in the EMR, as well as inter-office email, staff meetings, memos, and inter-office phone calls. Communication outside of the practice is almost exclusively letters in all three cases. Letter writing is the majority of work for secretaries as well as receptionists and some GPs. Any time that a primary care practice needs to communication information to another practice, secondary care, or another part of NHS it is done through a letter. In some instances this is also accomplished through a phone call depending on urgency of the matter and the recipient of the call. Also, in increasingly rare situations certain hospitals require a fax sent by facsimile machine. The third direction of communication is to the patient. This is done through phone calls, in some cases text messages, face-to-face while the patient is in clinic, and again through letters sent to the patient's home. These three transactional communication directions have proven to be a helpful shorthand in our analysis to show what other social actors are involved in the work of primary care, the tasks performed to communicate in and out of primary care, and lastly they show the challenges of automating certain work that happens to be a large bulk of the work in primary care.

\section{Conclusion}

How work will change in a future where automation and artificial intelligence are widespread is a timely and important conversation. It is a conversation that garners attention from academics, politicians, policy makers, and industry. The authors see the information field movement as an important community to bring nuance to the development, understanding, and use of automation and artificial intelligence in society, which arguably represent some of the highest-level interactions between humans and information to date. The early work presented in this paper attempts to contribute to this conversation through building a method and perspective of informing algorithms and technical development through a work practice perspective. It is our thesis that as tasks are automated, work sectors and occupations will undergo reconfiguration. In healthcare, these reconfigurations will not result in jobs disappearing wholesale, but the responsibilities, duties, and tasks performed by healthcare occupations will change (and indeed, arguably must change if the system is to survive the current socioeconomic pressures). We hope to anticipate some of this change through continued observation and analysis of the tasks that people perform and understanding, on a task based level, what can or cannot be automated. 


\section{Acknowledgements}

We would like to thank all of the participants for their time and expertise. We also thank The Health Foundation for their support of this work, award \# 7559.

\section{References}

1. Schmeiser, L.: Automation Invasion: Robots Are Coming For Your Job, http://observer.com/2017/03/automation-robots-american-jobs/, (2017).

2. Hammersley, B.: Think your job is safe from the robo-uprising? Think again, https://www.wired.co.uk/article/ai-robots-employment-jobs, (2016).

3. The Robot Revolution, http://content.time.com/time/covers/0,16641,19801208,00.html, (1980).

4. Anslow, L.: Robots have been about to take all the jobs for more than 200 years, https://timeline.com/robots-have-been-about-to-take-all-the-jobs-for-morethan-200-years-5c9c08a2f41d, (2016).

5. Bessen, J.E.: How Computer Automation Affects Occupations: Technology, Jobs, and Skills. SSRN Electron. J. (2015).

6. Frey, C.B., Osborne, M.A.: The future of employment: How susceptible are jobs to computerisation? Technol. Forecast. Soc. Change. 114, 254-280 (2017).

7. Corbin, J., Strauss, A.: Basics of qualitative research. Sage, Thousand Oaks, California (2008).

8. Glaser, B.G., Strauss, A.L.: The discovery of grounded theory. USA: Aldine, Chicago, IL (1967).

9. Bresnahan, T.F.: Computerisation and Wage Dispersion: An Analytical Reinterpretation. Econ. J. 109, 390-415 (1999).

10. Brynjolfsson, E., McAfee, A.: The second machine age : work, progress, and prosperity in a time of brilliant technologies. W. W. Norton \& Company (2014).

11. James Manyika, Michael Chui, Mehdi Miremadi, Jacques Bughin, Katy George, Paul Willmott, Martin Dewhurst: Harnessing automation for a future that works. (2017).

12. Ford, M.: The Rise of the Robots: Technology and the Threat of a Jobless Future. Oneworld Publications (2016).

13. Susskind, R.E., Susskind, D.: The future of the professions: how technology will transform the work of human experts. OUP Oxford (2017).

14. Baird, B., Charles, A., Honeyman, M., Maguire, D., Das, P.: Understanding pressures in general practice. , London, UK (2016).

15. Hopson, C.: The sate of the NHS provider sector. (2016).

16. Martin, S., Davies, E., Gershlick, B.: Under pressure: What the Commonwealth Fund's 2015 international survey of general practitioners means for the UK. (2016). 